\title{
Research on Calculation Method of Passing Capacity of High Speed Railway Station and Design of Simulation Process
}

\author{
Jiake Wu', Chao Zhang ${ }^{\mathrm{b}}$ \\ School of Traffic and Transportation, Beijing Jiaotong University, Beijing 100044, China. \\ a16120904@bjtu.edu.cn, b chzhang@bjtu.edu.cn
}

Keywords: High-speed railway, Station capacity, Comprehensive optimization, Simulation.

\begin{abstract}
The capacity of high-speed railway station has an important influence on the capacity of railway network. The existing capacity calculation methods cannot fully adapt to the characteristics of high-speed railway station operation. This paper analyses the influencing factors of the passing capacity of high-speed railway stations, studies the existing calculation methods and drawbacks, and focuses on the comprehensive optimization of common decision-making rules such as arrival-departure line usage rules and throat usage rules. The optimization model of high-speed railway station capacity is established, and the overall flow of computer simulation is designed.
\end{abstract}

\section{Introduction}

High-speed railway station is the key node of the transport organization of high-speed railway network and one of the factors determining the capacity of the line section. Its operational efficiency and capacity directly affect the transport capacity of the whole network.

Traditional station capacity includes throat capacity and arrival-departure line capacity ${ }^{[1]}$. At present, scholars at home and abroad have not yet formed an accurate and consistent definition of the passing capacity of high-speed railway stations. They mainly follow the definition of the passing capacity of ordinary railway stations, that is, the maximum number of trains that can be received and delivered in a day and night by a station under certain equipment conditions, with a certain train operation plan and reasonable operation organization method.

With the rapid development of high-speed railway in China, the research focus in the field of calculating the capacity of railway stations has gradually shifted to high-speed railway stations. For example, Li Qi ${ }^{[2]}$ constructed an optimization model for arrival and departure routes, but did not consider the coordinated optimization with throat route selection and only considered one objective; Qiao Ruijun et al. ${ }^{[3]}$ built a comprehensive optimization model based on the equilibrium of arrival and departure routes and the maximization of route preference. The objective weight assignment is subjective and can only get the local optimal solution of the problem. Liu Jie et al. ${ }^{[4]}$ established an optimization model for the comprehensive utilization of arrival-departure line and throat area of high-speed railway station, and solved it by genetic algorithm. The rationality of the occupancy cost of arrival-departure line needs to be discussed. Other scholars ${ }^{[5-8]}$ have some limitations on the integrity of the throat area to the departure line, the adaptability of the algorithm, and the stability of the operation. There are still important research values in the calculation, comprehensive optimization and Simulation of high-speed railway station capacity.

\section{Analysis of influencing factors on passing capacity of high speed railway station}

There are many factors affecting the capacity of high-speed railway stations. In 2004, the International Union of Railways (UIC) issued a capacity manual (code 406: capacity) ${ }^{[9]}$. It was proposed that the capacity is closely related to the capacity utilization scenario, which mainly includes the number of trains, train speed, operation chart structure, and stability requirements of operation chart. According to the relevant literature ${ }^{[5-7]}$, the main influencing factors of the capacity can be divided into two categories: static factors and dynamic factors, as shown in Table 1. 
Table 1 Influencing factors of passing capacity of high-speed railway station

\begin{tabular}{c|c|l}
\hline \multirow{2}{*}{ Static factors } & Station Technical Equipment & $\begin{array}{l}\text { Signal interlocking equipment, layout of station and } \\
\text { yard, type of throat turnout, etc. }\end{array}$ \\
\cline { 2 - 3 } $\begin{array}{c}\text { Station operation parameters } \\
\text { and rules }\end{array}$ & $\begin{array}{l}\text { Train interval time standard, train technical operation } \\
\text { flow, train technical operation time standard, etc }\end{array}$ \\
\hline \multirow{2}{*}{$\begin{array}{c}\text { factors } \\
\text { fanamic }\end{array}$} & $\begin{array}{c}\text { Demand } \\
\text { Station Operation } \\
\text { Organization Scheme }\end{array}$ & $\begin{array}{l}\text { Arrival and departure train structure, train operation } \\
\text { operation scheme, train receiving and departure } \\
\text { sequence scheme, etc. }\end{array}$ \\
\hline
\end{tabular}

Summarizes the following three outstanding differences:

First, the high-speed railway station does not need to handle the passenger train pick-up and hang-up and change trailers on the existing railway passenger station in the past, but only deals with the EMU train technical operation and passenger operation.

Secondly, the arrival and departure of freight trains, the transfer of passenger and freight trains and the locomotive entrance and exit sections are not required for high-speed railway. The occupancy time of fixed equipment for these operations, the hostile obstruction time for other turnout groups and the air cost coefficient introduced therefrom are not considered in the calculation of station capacity. Therefore, the high-speed railway station cannot simply imitate the existing station using fixed equipment occupancy time and space cost coefficient to calculate the carrying capacity.

Thirdly, the running of trains on high-speed railway is unbalanced. In order to facilitate passenger travel, there are obvious rush hours for receiving and departing trains at high-speed railway stations. The capacity of arrival and departure lines and throats of stations is tense, which is in sharp contrast with the surplus capacity of peak hours. Therefore, the peak time is the bottleneck stage to limit the capacity of the station. The traditional calculation method is to calculate the receiving capacity of the station in a full day, which cannot reflect the actual peak capacity of the station, and has great limitations.

\section{Analysis on the calculation method of passing capacity of high-speed railway station in China}

In the Code for Design of High-Speed Railway (TB10621-2014), it is pointed out that the calculation of station capacity should be based on the maximum utilization of station parallel routes and the rational use of station lanes under certain conditions of station equipment configuration and operation organization. The throat of station, the capacity of arrival and departure routes and the requirement of arrival and departure routes during peak hours are calculated respectively ${ }^{[10]}$. The temporary calculation method of high-speed railway station capacity is given in the code, and it is pointed out that it needs to be determined after thorough study combined with the characteristics of high-speed railway in China. The specific calculation methods for the capacity of high-speed railway stations in China are as follows:

\subsection{Throat capacity calculation}

Throat capacity in all directions refers to the sum of the capacity of each turnout group. Firstly, the turnout groups are divided, and then the utilization ratio of each turnout group is calculated separately. Then, the receiving and departing capacity of each turnout group under this utilization ratio is calculated. The total capacity is obtained by summing up the receiving and departing capacity of all turnout groups in this direction.

a. Utilization rate of throat turnout group's full-day capacity:

$$
K=\frac{T-\sum t_{\text {fixed }}}{\left(1440-T_{\text {stop }}-\sum t_{\text {fixed }}\right)\left(1-\gamma_{\text {waste }}\right)}
$$


In formula, $T$-Total occupancy time (min) of pharynx and larynx switch group; $\gamma_{\text {waste }}$-The empty fee coefficient of the pharynx-throat switch group is $0.2-0.3 ; T_{\text {stop }}$ - The time (min) when the station stops receiving and departing buses within one day and night; $\sum t_{\text {fixed }}$-Fixed operation takes up the total time (min) of the pharynx and throat turnout group.

b. Utilization rate of throat turnout capacity during peak period:

$$
K=\frac{T-\sum t_{\text {fixed }}}{\left(T_{\text {peak }}-\sum t_{\text {fixed }}\right)\left(1-\gamma_{\text {waste }}\right)}
$$

c. Throat capacity is calculated by the following formula:

$$
N_{\text {arrive }}^{i}=\frac{n_{\text {arrive }}^{i}}{K}, \quad N_{\text {depart }}^{i}=\frac{n_{\text {depart }}^{i}}{K}
$$

\subsection{Calculation of Passing Capacity of Arrival-departure Line}

At present, the capacity of arrival and departure lines of high-speed railway stations is calculated by direct calculation method.

Full-time to-departure line Passing capacity:

$$
N_{p}=\frac{M_{p}\left(1440-T_{\text {stop }}\right)\left(1-\gamma_{\text {waste }}\right)}{t_{\text {average }}} \times K
$$

The main drawbacks of the above calculation methods are as follows:

Firstly, following the traditional calculation method, this method is based on the balanced distribution of all kinds of train flow from the beginning to the end, that is, the capacity and utilization ratio are linearly related. It cannot accurately reflect the randomness and imbalance of high-speed railway stations.

Secondly, there is subjectivity in the selection of relevant parameters, especially the empty fee coefficient. With a certain approximation and idealization, the impact of empty time caused by different factors on the capacity varies.

Thirdly, in the calculation, it is assumed that each arrival and departure line is completely balanced, which cannot reflect the actual occupancy of arrival and departure lines, thus distorting the calculation results.

Fourthly, it cannot reflect the influence of different speed levels and different types of operation on the arrival and departure line capacity.

In recent years, many scholars ${ }^{[11]}$ have adopted the idea of comprehensive optimization to study the capacity of high-speed railway, and transformed the capacity solving into a multi-objective and multi-point decision-making problem. Optimize the summary of commonly used decision rules as shown in Table 2.

How to use the above decision rules and establish a suitable comprehensive optimization model is the difficulty of the study. On this basis, this paper establishes a comprehensive optimization model for the capacity of high-speed railway stations as follows.

\section{Optimization model}

\subsection{Model Foundation and Hypothesis}

It is assumed that all train speed classes are the same as those of models, given the information of station type, station track information, turnout and signal route data, equipment connection relationship, train diagram, traffic flow composition, station operation time standard, safety interval time standard and construction skylight time standard. 
Table 2 Common Decision Rules

\begin{tabular}{|c|c|c|}
\hline Decision content & Decision rule & Decision-making/assessment indicators \\
\hline \multirow{3}{*}{$\begin{array}{l}\text { Types of train Flow } \\
\text { Composition }\end{array}$} & Train type & Speed, Model \\
\hline & Train operation types & Pass, Start, End, Return \\
\hline & $\begin{array}{c}\text { Orientation of arrival and } \\
\text { departure of train }\end{array}$ & Receiving in the same direction and in different directions \\
\hline \multirow{3}{*}{$\begin{array}{l}\text { Rules for the Use of } \\
\text { Track }\end{array}$} & Equilibrium Use rule & $D(t)=\sum_{i=1}^{M}\left(\sum_{j=1}^{s_{i}}\left|t_{i j}^{\prime \prime}-t_{i j}^{\prime}\right|-\frac{1}{M} \sum_{i=1}^{M} \sum_{j=1}^{s_{i}}\left|t_{i j}^{\prime \prime}-t_{i j}^{i}\right|\right)^{2}$ \\
\hline & Compact use rule & $\begin{array}{l}F(t)=\sum_{i=1}^{M}\left(\sum_{j=2}^{s_{i}}\left|t_{i j}^{\prime}-t_{i(j-1)}^{\prime \prime}\right|+\left|t_{i 1}^{\prime}-T_{\text {start }}\right|\right) \\
D(t)^{[16]}\end{array}$ \\
\hline & $\begin{array}{l}\text { Minimum job cross-interference } \\
\text { use rule }\end{array}$ & $F(r)=\sum_{r \in R}\left\{1-\sum_{l=1}^{L_{r}}\left[\sum_{i 1 \in D_{j 1}} x_{j 111} \cdot \sum_{i 2 \in D_{j 2}} x_{j 2 i 2}\right]\right\}$ \\
\hline \multirow{3}{*}{$\begin{array}{l}\text { Rules for the Use of } \\
\text { Throat }\end{array}$} & Maximum Parallel Route Rule & $F(n)=\operatorname{num}\left(P_{l}\right)$ \\
\hline & $\begin{array}{l}\text { Maximum Probability Route } \\
\text { Rule }\end{array}$ & $F(n)=\sum_{k=1}^{L} n_{k l}$ \\
\hline & Minimum route rule & $F(t)=\sum_{j=1}^{J} \sum_{l \in L_{i}} y_{j l} t_{j l}$ \\
\hline
\end{tabular}

\subsection{Variable settings}

Suppose that the number of trains receiving and departing from high-speed railway stations in a certain period of time is $m$. According to arrival order number it as $i$. The train assembly is $I=\{1,2, \cdots, i, \cdots, m\}$; The set of arrival and departure track: $J=\{1,2, \cdots, j \cdots, n\}$; The set of routes from train $i$ to Arrive line $j$ is $A_{i j}, a_{i j}^{k}$ indicates the choice of route $k$ for train $i$ to connect to Arrive line $j, a_{i j}^{k} \in A_{i j}, k \leq p$; The set of routes from train $i$ to depart line $j$ is $D_{i j}, d_{i j}^{k}$ indicates the choice of route $k$ for train $i$ to connect to departure line $j, d_{i j}^{k} \in D_{i j}, k \leq q$; Using $t_{i j}^{a}, t_{i j}^{d}$ represent the time of arrival and departure of trains.

Decision variables have three $0-1$ variables, $x_{i j}$ : the line $j$ occupied by train $\mathrm{i} ; y_{i j}^{k}$ : The train $i$ access line $j$ chooses the $k$ access route. $z_{i j}^{k}$ : Train $i$ access line $j$ chooses the $k$ departure route.

\subsection{Objective function}

a. The matching between arrival and departure line occupancy and train is the most reasonable, and the matching degree is reflected by the cost of arrival and departure line occupancy.

$$
\min z_{1}=\sum_{i} \sum_{j} c_{i j} x_{i j}
$$

$c_{i j}$ is the cost of occupying the line, which is related to the type of trains and operation time.

b. Choose the shorter route with fewer turnouts to finish the receiving and departing operation, and adopt the route occupancy cost to reflect the merits and demerits of the scheme. The objective function is as follows:

$$
\min z_{2}=\sum_{i} \sum_{j} \alpha_{j}^{k} y_{i j}^{k}+\sum_{i} \sum_{j} \beta_{j}^{k} z_{i j}^{k}
$$

$\alpha_{j}^{k}, \beta_{j}^{k}$ is the cost, which is related to the length of the route, the running time of the train, the number and location of the turnout. 


\subsection{Constraint condition}

a. Trains can only choose one arrival and departure line for operation.

$$
\sum_{j=1}^{n} x_{i j}=1, \forall i=I
$$

b. Arrival and departure lines effectively meet train access conditions.

$$
\sum_{j=1}^{n}\left[x_{i j}\left(L_{j}-l_{i}\right)\right] \geq 0, \forall i \in I
$$

c. Trains can only choose one arrive and departure route.

$$
\sum_{j=1}^{n} \sum_{k=1}^{p} y_{i j}^{k}=1, \forall i \in I \sum_{j=1}^{n} \sum_{k=1}^{q} z_{i j}^{k}=1, \forall i \in I
$$

d. Minimum safety interval must be satisfied between adjacent train operations reaching the same arrival and departure line.

$$
t_{i j}^{a}-t_{i j}^{d}<T_{\text {safe }}
$$

For this kind of problem, there are two kinds of solutions: one is to transform the multi-objective problem into the single-objective problem, but it is difficult to determine the weight and other parameters in the process of transformation, which are greatly influenced by human factors; the other is to solve the model by using heuristic algorithm, and to find the optimal solution set with convergence quickly and comprehensively is the difficulty of solving the problem. The improved particle swarm optimization algorithm, genetic algorithm and so on can be used. Tabu search and other algorithms to solve, the design of a reasonable optimization path and effectiveness is the key, this paper will not do in-depth study.

\section{Design of simulation process}

Based on the simulation of station operation process, under the conditions of meeting various operation rules and time standards, train operation time and path are randomly arranged, and the station capacity composition under different capacity utilization scenarios is calculated. Despite the randomness of the simulation results, the approximate maximum number of train operations at the station can be obtained through multiple simulation tests. The simulation process design is shown in Figure 1 below.

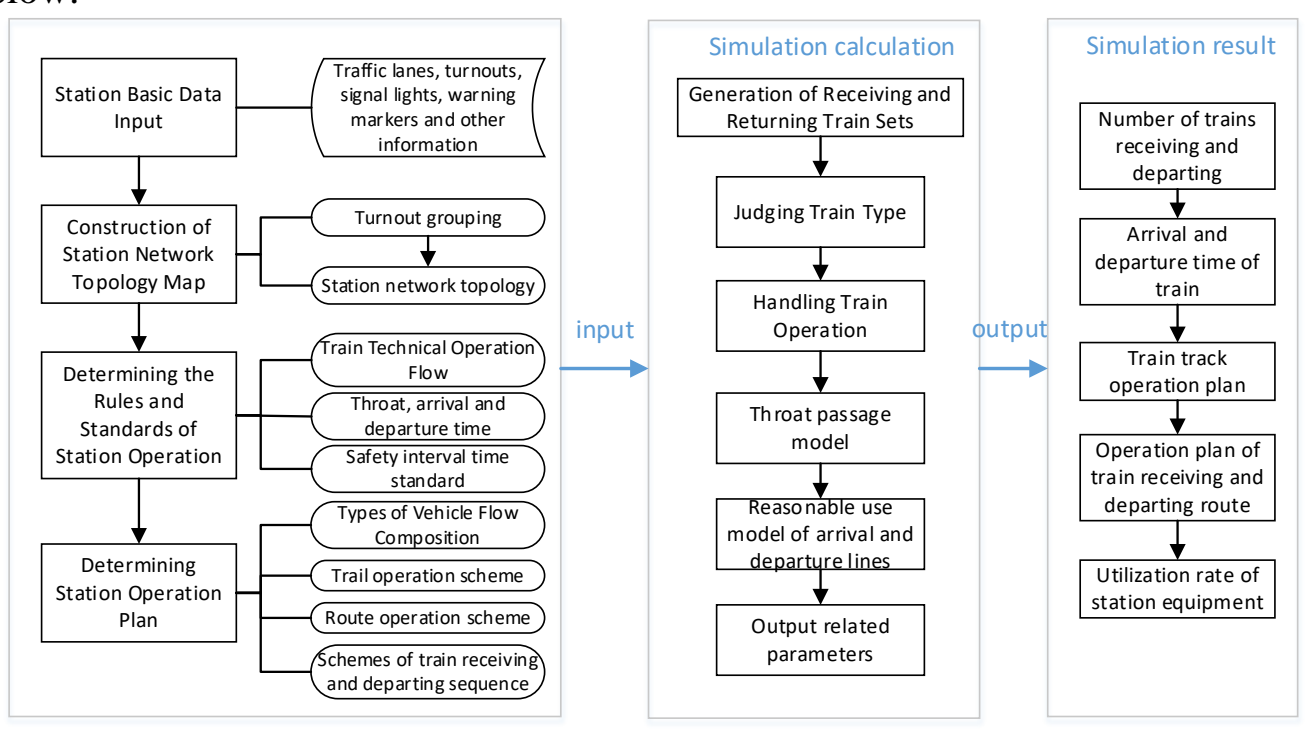

Fig. 1 The simulation process 


\section{Summary}

High-speed railway station has special operation characteristics, so the traditional calculation method of station capacity is no longer applicable. The existing calculation methods for the capacity of high-speed railway stations cannot fully meet the actual needs, but still follow the classical calculation methods based on the utilization rate calculation method, aiming at balancing the use of station resources, which has obvious disadvantages. This paper puts forward a comprehensive optimization model of high-speed railway station throughput capacity by analyzing the influencing factors and decision rules, and designs the flow of computer simulation algorithm for high-speed railway station operation, which has certain reference value and reference significance for computer simulation research.

\section{References}

[1] Yang Hao. Histology of Railway Transportation [M]. Beijing: China Railway Press, 2013.

[2] Li Qi. Study on the optimization of arrival and departure line operation of large high-speed railway passenger stations [D]. Chengdu: Southwest Jiaotong University, 2012.

[3] Qiao Ruijun, Zhu Xiaoning, Qian Jifeng. Optimizing the route selection of passenger dedicated line station reception and departure [J]. Logistics technology, 2012, 31 (9): 201-204.

[4] Liu Jie, Yin Yong, Gan Zhiliang. Comprehensive optimization of throat area and arrival-departure line of high-speed railway station [J]. Transportation system engineering and information, 2018,18(1): 193-199

[5] Wendong. Passenger Station Capacity Study of Passenger Dedicated Line [D]. Southwest Jiaotong University, 2006.

[6] Liu Min, Han Baoming, Li Dewei. Calculating and evaluating the capacity of high-speed railway station [J]. Journal of Railway, 2012, (04):9-15.

[7] Chen Tao. Research on the theory and method of calculating the capacity of high-speed railway station [D]. Southwest Jiaotong University, 2016.

[8] Li Xiaojuan. Research on calculation method of high-speed railway capacity and reliability evaluation method [D]. Beijing Jiaotong University, 2016.

[9] UIC.UIC code 406: Capacity [S]. Paris-France: UIC, 2004.

[10] State Railway Administration. Design Code for High Speed Railway (TBl0621-2014) [M]. Beijing: China Railway Publishing House. 2017

[11] Shi Feng, Xie Chunong, Yu Guifang, et al. [J]. Optimizing method of route arrangement in throat area of railway passenger station [J]. Journal of Railway, 2004, 8, 26 (4):5-9. 